\title{
Association between Vitiligo and Thyroid Autoimmunity in Jordanian Population
}

\author{
Alawneh $\mathbf{S}^{1^{*}}$, Ayman Abu Awad ${ }^{2}$, Mamoon Athamneh ${ }^{2}$, Salim Abdel Rahman ${ }^{3}$, Mohammad Abu-Zaid $^{2}$, Omema D Abu-Alsondos ${ }^{2}$ and Nedal Alnawaiseh ${ }^{4}$ \\ ${ }^{1}$ Faculity of Medicine, Immam Abdul Rahman Ibn Faisel University, Kingdom of Saudi Arabia \\ ${ }^{2}$ King Hussein Medical Center, Department of Dermatology, Amman, Jordan \\ ${ }^{3}$ Department of Biotechnology, Hashemite University, Zarqa, Jordan \\ ${ }^{4}$ Department of Dermatology, Medical School, MutahUniversity, Karak, Jordan
}

"Corresponding author: Alawneh S, Faculity of Medicine, Immam Abdul Rahman Ibn Faisel University, Kingdom of Saudi Arabia, E-mail: sammiomar@hotmail.com Received date: March 18, 2018; Accepted date: April 07, 2018; Published date: April 12, 2018

Copyright: $\odot 2018$ Alawneh S, et al. This is an open-access article distributed under the terms of the Creative Commons Attribution License, which permits unrestricted use, distribution, and reproduction in any medium, provided the original author and source are credited.

\begin{abstract}
Vitiligo is considered to be the most commonly acquired hypomelanosis. In this study, we investigated some of the issues with regards to Vitiligo and its association with autoimmune thyroiditis. In this case-control study, thyroxine (FT4), triiodothyronine (FT3), and thyroid stimulating hormone (TSH) were measured in 130 patients with vitiligo and in 99 healthy volunteers. Also, we compared the frequency of thyroid autoantibodies (anti-Tg, thyroid peroxidase antibody, and anti-TPO) in the same groups.
\end{abstract}

Results: Showed that thyroid functional abnormalities were found in $6(18.18 \%)$ patients. Anti-Tg and anti-TPO were positive in $115(88.5 \%)$ patients respectively. In the control group, only nine subjects $(9.1 \%)$ had abnormalities in thyroid hormonal condition, and two subjects had positive thyroid autoantibodies compared with the control group, and the frequency of both anti-Tg and anti-TPO was significantly higher in those with vitiligo.

Conclusion: This study showed a significant association between vitiligo and thyroid autoimmunity and the tests to detect thyroid autoantibodies that are appropriate in patients with vitiligo.

Keywords: Hypomelanosis; Thyroxine; Autoantibodies; Autoimmunity; Thyroid

\section{Introduction}

Vitiligo is considered to be the most commonly acquired hypomelanosis [1]. In this study, we will investigate the association of Vitiligo with Autoimmune Thyroiditis by indicating the epidemiological profile of this skin disease, which will raise the attentiveness of physicians about the need for a more careful assessment of patients with vitiligo and to trace their epidemiological profile in order to estimate the association between vitiligo and thyroid diseases among Jordanian population. Also, in this study, we will compare our results with the current and previous studies conducted in different areas around the world. The other purpose of this investigation was to study the occurrence of concomitant autoimmune diseases in Jordanian patients with generalized or localized vitiligo as well as their families.

\section{Patients and Methods}

The study included 130 patients with vitiligo, 71 female and 59 male, with mean age 26.54 ( \pm 14.87$)$ years. Of them, there were $89(68.9 \%)$ patients with generalized vitiligo and $40(31.1 \%)$ with localized form of the disease. A detailed history and examination were conducted for all study subjects, including the patient's age, age at onset, duration of disease, associated diseases, history of thyroid disorders, and the extent and severity of the disease. The diagnosis of vitiligo was made on clinical grounds according to standard criteria. The control group consisted of 99 volunteers, 20 (20.2\%) female, and 79 (79.8\%) male, with mean age $27.16( \pm 14.41)$ years. Blood samples were withdrawn and a physical examination and thyroid sonography were performed by Dermatologist at King Hussein Medical Center (KHMC) in Amman, Jordan. All subjects gave their informed consent in accordance with the requirements of the Institutional Ethics Committee.

Thyroid autoantibodies (thyroglobulin antibody, anti-Tg and thyroid peroxidase antibody, anti-TPO) and thyroid hormones (thyroxine (T4), tri-iodthyronine (T3), and thyroid stimulating hormone (TSH) were measured in all subjects. Total T4 (normal range: $70-180 \mathrm{nmol} / \mathrm{L}$ ) and total T3 (normal range: $1.3-3.3 \mathrm{nmol} / \mathrm{L}$ ) were measured by the use of radioimmunoassay (RIA); TSH (normal range: 0.3-4.2 $\mathrm{mL} \mathrm{U/L}$ ) was determined by the use of immunoradiometric assay (IRMA) (BRAHMS Aktiengesellschaft, Hennigsdorf, Germany). Serum levels of anti-Tg (threshold value: $25 \mathrm{IU} / \mathrm{mL}$ ) and anti-TPO (borderline value: $34 \mathrm{IU} / \mathrm{mL}$ ) were measured by the use of Electrochemiluminescence immunoassay (ECLIA) based on standard protocols (COBAS, Roche Diagnostics GmbH, Germany). The upper limit of autoantibody was determined by the laboratory.

The collected data were analyzed by SPSS version-16 in vitiligo patients and subgroups according to their gender, age, extent, and duration of the disease, when compared with the control group.

Statistical comparisons were performed using chi-square test. Data were considered statistically significant at 0.05 levels. 
Citation: Alawneh S, Awad AA, Athamneh M, Abdelrahman S, Abu-Zaid M, et al. (2018) Association between Vitiligo and Thyroid Autoimmunity in Jordanian Population. J Immuno Biol 3: 138. doi:10.4172/2476-1966.1000138

Page 2 of 3

\section{Results}

We performed a case control study in 130 consecutive patients with vitiligo and 99 age and sex-matched controls. Demographic data of patients and controls are shown in Table 1 . The mean (SD) age of the patients and control groups was $26.54( \pm 14.87)$ and $27.16( \pm 14.81)$ correspondingly. The duration of vitiligo ranged from 1.8 to 139.6 months. Eighty nine patients had generalized, and forty patients had localized vitiligo (Table 2). Thyroid functional abnormalities were found in $115(88.5 \%)$ patients for both anti-Tg and anti-TPO. In the control group, only nine $(9.1 \%)$ and eleven $(11.1 \%)$ subject had abnormalities in hormonal status for anti-Tg and anti-TPO, respectively.

\begin{tabular}{|l|l|l|l|l|}
\hline & Patient vs. Control & \multicolumn{2}{l|}{ Total } & \multicolumn{2}{|l|}{ P-value } \\
\hline & Patients & Control & \multicolumn{2}{|l|}{} \\
\hline Female, $\mathrm{n}(\%)$ & $71(54.6 \%)$ & $21(20.2 \%)$ & $91(39.7 \%)$ & $<0.001$ \\
\hline Male, $\mathrm{n}(\%)$ & $59(45.4 \%)$ & $79(79.8 \%)$ & $138(60.3 \%)$ & $2-61$ \\
\hline Age Range, Years & $4-58$ & $2-61$ & $26.81(14.81)$ & \\
\hline Age, Mean Years \pm SD & $26.54(14.87)$ & $27.16(14.81)$ & $261)$ & \\
\hline
\end{tabular}

Table 1: Demographic data of patients (Vitiligo group) and (Control group).

\begin{tabular}{|l|l|}
\hline Mean age of onset \pm SD (year) & $20.66 \pm 15.57$ \\
\hline Age of onset range (year) & $9-53$ \\
\hline Mean duration \pm (SD) (month) & $70.53 \pm 68.73$ \\
\hline Duration Range (month) & $0.5-252$ \\
\hline Type of vitiligo $\mathbf{n},(\%)$ & $89(68.9)$ \\
\hline Generalized & $40(31.1)$ \\
\hline Localized & \\
\hline
\end{tabular}

Table 2: Clinical characteristics of patients with vitiligo.

In the patients with vitiligo, anti-Tg titers ranges from 10 to more than $500 \mathrm{IU} / \mathrm{mL}$ and anti-TPO antibody titers from 10 to $517 \mathrm{IU} / \mathrm{mL}$. In the control group, anti-Tg titers were ranging from 6 to $82 \mathrm{IU} / \mathrm{mL}$, and anti-TPO antibody titers were from 10 to $88 \mathrm{IU} / \mathrm{mL}$. Anti-Tg antibody in 44 (33.8\%) patients, anti-TPO antibody in 102 (78.5\%) and both anti-Tg and anti-TPO antibodies in $43(33.1 \%)$ were higher than the normal antibody titers. Reference normal ranges used were less than $23 \mathrm{IU} / \mathrm{mL}$ for anti-Tg and less than $25 \mathrm{IU} / \mathrm{mL}$ for anti-TPO. In the control group, zero (0\%) had positive anti-Tg and two volunteer $(2.02 \%)$ had positive anti-TPO. The incidence of thyroid autoantibodies was significantly higher in vitiligo patients when compared to the control group (Table 3) (Same thing applied for males and females). Statistically significant differenceswere also found in the values of anti-Tg and anti-TPO with generalized and localized vitiligo (Table 3).

\begin{tabular}{|l|l|l|l|l|}
\hline & \multicolumn{2}{|l|}{ Anti-Tg (threshold value 23 IU/mL) } & \multicolumn{2}{l|}{ Anti-TPO (threshold value 25 IU/mL) } \\
\hline Group & Negative $\mathrm{n}(\%)$ & Positive $\mathrm{n}(\%)$ & Negative $\mathrm{n}(\%)$ & Positive $\mathrm{n}(\%)$ \\
\hline Vitiligo & $15(11.5)$ & $115(88.5)$ & $15(11.5)$ & $115(88.5)$ \\
\hline Control & $90(90.9)$ & $9(9.1)$ & $88(88.9)$ & $11(11.1)$ \\
\hline Total & $105(45.9)$ & $124(54.1)$ & $103(45.0)$ & $126(55.0)$ \\
\hline $\mathrm{X} 2, \mathrm{P}, \mathrm{df}^{*}$ & $\mathrm{X} 2=4.19, \mathrm{P}=0.040, \mathrm{df}=1$ & $\mathrm{X}=4.21, \mathrm{P}=.040, \mathrm{df}=1$ & \\
\hline
\end{tabular}

Table 3: Frequency of thyroid autoantibodies in the study group; ${ }^{\star} \mathrm{df}=$ degree of freedom.

A Chi-square test for independence (with Yates Continuity Correction) indicated significant association between higher values of anti-Tg (values more than $23 \mathrm{IU} / \mathrm{ml}$ ) and vitiligo.
A Chi-square test for independence (with Yates Continuity Correction) indicated significant associations between higher values of anti-TPO (values more than $25 \mathrm{IU} / \mathrm{ml}$ ) and vitiligo. 


\begin{tabular}{|c|c|c|c|c|c|c|}
\hline Group & Case & $\begin{array}{l}\text { Positiv } \\
\text { e TG- } \\
\text { Ab (\%) }\end{array}$ & $\begin{array}{l}\text { Positiv } \\
\text { e TPO } \\
\text { Ab (\%) }\end{array}$ & FT3 & FT4 & TSH \\
\hline Control & 99 & -9.1 & 11.1 & $3.87 \pm 0.59$ & $1.25 \pm 0.21$ & $2.61 \pm 0.13$ \\
\hline Vitiligo & 130 & $88.5^{\star}$ & $88.5^{*}$ & $\begin{array}{l}6.16 \\
2.81^{* *}\end{array} \quad \pm$ & $\begin{array}{ll}2.56 & \pm \\
1.62^{* *}\end{array}$ & $\begin{array}{l}7.59 \\
0.99^{* *}\end{array}$ \\
\hline
\end{tabular}

Table 4: Comparison of altered thyroid parameters between Vitiligo and control groups;Compared with control group; ${ }^{*} \mathrm{p}<0.05,{ }^{* *} \mathrm{p}<0.001$.

\section{Discussion}

Vitiligo is a mal-pigmentation disorder which occurs due to the damage of melanocytes [1,2]. However, its etiology has not yet been fully resolved [3]. For that reason, there are many theories about its etiology, including autoimmune, genetic, autotoxic, and neural [4]. The autoimmune etiology seems to be most reasonable, in which the destruction of melanocytes is secondary to autoantibodies, which are related to the extent and activity of the disease [5]. This theory is based on the observations made of the simultaneous occurrence of vitiligo and autoimmune diseases, such as pernicious anemia, rheumatoid arthritis (RA), diabetes mellitus, thyroiditis, alopecia areata, and others $[6,7]$. Vitiligo is most commonly associated with autoimmune thyroid disease [8].

In this study, we have shown an association between vitiligo and thyroid autoimmunity markers, such as T3,T4,TSH, anti-Tg, and antiTPO. Here, our results show signficant differences in the level of these markers between the control group and normal group (Table 4).

The occurence of TG-Ab and TPO-Ab was higher in vitiligo patients than in the control subjects $(\chi 2=9.4$ and $8.9, \mathrm{P}=0.05$ and $0.003)$. The TSH levels were significantly higher in vitiligo patients than in the control subjects $(\mathrm{P}<0.05)$. The FT3 and FT4 levels were also slightly higher in the vitiligo patients. What is more, patients with positive TG-Ab and TPO-Ab had vitiligo vulgaris. Among 13 vitiligo patients with elevated TSH, 12 (92.3\%) were patients with vitiligo vulgaris and $1(7.6 \%)$ had segmental vitiligo (Table 1$)$.

The difference of the frequency of anti-TPO was not significant with regards to the duration and extent of vitiligo. In addition, there was no significant difference in the levels of free T3, free T4, and TSH in the vitiligo patients when compared to the control group (Table 2).

This has a great impact, since this association can be used in the future to cure vitiligo by targeting thyroid autoimmunity markers. The same results were found for both males and females, which indicated towards the generalized conclusion of the association between Thyroid Autoimmunity markers and vitiligo [9-11]. Other studies has had to be done to investigate the association between vitiligo and other autoimmune diseases, and many of them showed significant associations between them $[9,10]$. Doing such studies is of great importance in Jordan, as this will enable us to benefit from medications discovered to cure vitiligo and other autoimmune diseases harmonious
The occurence of TG-Ab and TPO-Ab was higher in vitiligo patients than in control subjects $(\chi 2=9.4$ and $8.9, \mathrm{P}=0.002$ and 0.003$)$. The TSH levels were significantly higher in the vitiligo patients than in the control subjects $(\mathrm{P}=0.045)$. The FT3 and FT4 levels were also slightly higher in the vitiligo patients. What is more, patients with positive TG-Ab and TPO-Ab had vitiligo vulgaris. Amongst the 13 vitiligo patients with elevated TSH, $12(92.3 \%)$ were those with vitiligo vulgaris and $1(7.6 \%)$ had segmental vitiligo (Table 1$)$.

\section{Conclusion}

Among the Jordanian vitiligo patients, there is a subgroup with strong evidence of determined susceptibility to not only vitiligo, but also to autoimmune thyroid disease and the presence of TG-Ab and $\mathrm{TPO}-\mathrm{Ab}$ are the major markers for thyroid autoimmunity. So, by monitoring TG-Ab and TPO-Ab, we can understand the characteristics and extent of AITD [12]. Thus, it seems plausible to screen for thyroid antibodies in vitiligo.

\section{References}

1. Birlea SA, Fain PR, Spritz RA (2008) A Romanian population isolate with high frequency of vitiligo and associated autoimmune diseases. Arch Dermatol 144: 310-316.

2. Boissy RE, Nordlund JJ (2011) Vitiligo: current medical and scientific understanding. G Ital Dermatol Venereol 146: 69-75.

3. Carle A, Laurberg P, Knudsen N, Perrild H, Ovesen L, et al. (2006) Thyroid peroxidase and thyroglobulin auto-antibodies in patients with newly diagnosed overt hypothyroidism. Autoimmunity 39: 497-503.

4. Iacovelli P, Sinagra JLM, Vidolin AP, Marenda S, Capitanio B, et al. (2005) Relevance of thyroiditis and of other autoimmune diseases in children with Vitiligo. Dermatology 210: 26-30.

5. Kovacs SO (1998) Vitiligo. J Am Acad Dermatol 38: 647-666.

6. Laberge G, Mailloux CM, Gowan K, Holland P, Bennett DC, et al. (2005) Early disease onset and increased risk of other autoimmune disease in familial generalized vitiligo. Pigment Cell Res 18: 300-305.

7. Liao EY (2001) Beijing, China: People's Health Press. Endocrinology Pp: 614-647.

8. Kakourou T, Kanaka-Gantenbein C, Papadopoulou A, Kaloumenou E, Chrousos GP (2005) Increased prevalence of chronic autoimmune (Hashimoto's) thyroiditis in children and adolescents with vitiligo. J Am Acad Dermatol 53: 220-223.

9. Kasumagic-Halilovic E, Prohic A, Begovic B, Ovcina-Kurtovic N (2011) Association between Vitiligo and Thyroid Autoimmunity. J Thyroid Res 2011: 938257

10. Radetti G, Gottardi E, Bona G, Corrias A, Salardi S, et al. (2006) The natural history of euthyroid Hashimoto's thyroiditis in children. J Pediatr 149: 827-832.

11. Dave S, D'Souza M, Thappa DM, Reddy KS, Bobby ZB (2003) High frequency of thyroid dysfunction in Indian patients with vitiligo. Indian J Dermatol 48: 68-72.

12. Zettinig G, Tanew A, Fischer G, Mayr W, Dudczak R, et al. (2003) Autoimmune diseases in vitiligo: Do anti-nuclear antibodies decrease thyroid volume? Clin Exp Immunol 131: 347-354. 